\title{
Statistical transmutation of quantum bosonic strings coupled to general four-dimensional Chern-Simons theory
}

\author{
J. Barcelos-Netof and E.C. Marinof \\ Instituto de Física \\ Universidade Federal do Rio de Janeiro, \\ RJ 21945-970 - Brazil
}

(Dated: November 12, 2018)

\begin{abstract}
A bosonic string coupled to the generalized Chern-Simons theory in $3+1 \mathrm{D}$ acquires a magnetic field along itself, when it is closed, and a topological charge at its extremity, when it is open. We construct the creation operators for the full quantum field states associated to these strings and determine the dual algebra satisfied by them. We show that the creation operator fo the composite state of a quantum closed bosonic string, bearing a magnetic flux, and a topologically charged open bosonic string, possesses generalized statistics. The relation of our results with previous approaches to the problem is also established.

PACS numbers: 11.10.Ef, 11.15.-q, 11.25.-w, 05.90.+m
\end{abstract}

\section{INTRODUCTION}

The obtainment of a full quantum field theory of strings would require the construction of the complete creation operator for the string and the evaluation of its correlation functions. Nevertheless, a full quantum description of the statistical transmutation undergone by bosonic strings when coupled to certain fields, surprisingly, can be achieved at an intermediate stage, where the fields attached to the string by the interaction are fully quantized. The achievement of this description, which may be considered as a partial field quantization of the bosonic string is the main purpose of this work.

Statistical transmutation of bosonic strings has been recently investigated in various different frameworks 过, 2, 3, 4]. It has been shown in particular [2] that a closed spatial string bearing a magnetic flux and moving in the presence of the field of a classical topological charge would undergo statistical transmutation. Here we provide the full quantum description of this fact in the framework of the generalized Chern-Simons (CS) theory in $3+1$ dimensions. This theory has been shown to belong to a hierarchy of theories, related by dimensional reduction, which contains the usual CS Lagrangian in $2+1 \mathrm{D}$ as a particular case [5]. We start from the observation that a bosonic string coupled to this theory bears a magnetic flux along itself, whenever it is closed and a topological charge at its extremity, whenever it is open. We then explicitly construct the operators creating the full quantum field states, which are attached to the string through the coupling. The creation operator for the magnetic field along the string is basically the same one considered previously in the framework of the BFtheory 判. The topological charge quantum field creation

\footnotetext{
*Electronic address: barcelos@if.ufri.br
}

${ }^{\dagger}$ Electronic address: marino@if.ufrj.br operator, however, is specific for the CS-theory. We show that these operators indeed create eigenstates of the corresponding appropriate operators with the correct eigenfield configurations. We then show that the magnetic field and topological charge creation operators satisfy a dual algebra, which implies that their product possesses generalized statistics. We also show that our result is the operator version of the statistical transmutation of bosonic Nambu-Goto strings coupled to CS theory, observed in [1] within the functional integral framework.

We mention that there is another CS term at $D=3+1$ [5] involving a scalar and rank three gauge fields. However, this term is not related to statistical transmutation because there is no charge-flux system associated to it.

The procedure we have presented in this paper can be naturally extended to higher spacetime dimensions, where statistical transmutation of membranes coupled to gauge fields of rank higher than two should occur [6].

\section{CHERN-SIMONS THEORY IN 3+1D AND ITS COUPLING TO THE STRING}

Chern-Simons theory in $3+1 \mathrm{D}$ involves a gauge vector field $A_{\mu}$ as well as a gauge tensor field $B_{\mu \nu}$ (KalbRamond) and is characterized by the Lagrangian [5, 7]

$$
\mathcal{L}_{C S}=\frac{1}{2} \epsilon^{\mu \nu \alpha \beta} A_{\mu} \partial_{\nu} B_{\alpha \beta} .
$$

Consider now a point particle, described by the current $j^{\mu}$ and a string placed at the curve $C$ and associated to the current density

$$
J^{\mu \nu}=\int_{S(C)} d^{2} \xi^{\mu \nu} \delta(x-\xi),
$$

where $S(C)$ is the universe-sheet of the string. We may couple these to the Chern-Simons Lagrangian in the usual way, namely, 


$$
\mathcal{L}=\frac{1}{2} \epsilon^{\mu \nu \alpha \beta} A_{\mu} \partial_{\nu} B_{\alpha \beta}-\frac{1}{2} J^{\mu \nu} B_{\mu \nu}-j^{\mu} A_{\mu} .
$$

The field equations associated to 2.3 are

$$
\begin{aligned}
& J^{\mu \nu}=\epsilon^{\mu \nu \alpha \beta} \partial_{\alpha} A_{\beta} \\
& j^{\mu}=\epsilon^{\mu \nu \alpha \beta} \partial_{\nu} B_{\alpha \beta} .
\end{aligned}
$$

We see that the string density along direction $i$ is given by

$$
J^{0 i}=\epsilon^{i j k} \partial_{j} A_{k}=\mathcal{B}^{i}
$$

implying the existence of a magnetic field $\overrightarrow{\mathcal{B}}$ along the string. Note, however, that since magnetic field lines should be closed, only closed strings can bear a magnetic flux. Also the second field equation means that $j^{\mu}$ is identified with the topological current, implying that a topological charge is associated to the particle coupled to $A_{\mu}$. A very interesting case is the one in which we take

$$
j^{\mu}=\oint_{C} d \xi^{\mu} \delta^{4}(x-\xi)
$$

where $C$ is the border of the universe-sheet of the string. For a closed spatial string $j^{0}=0$, implying that this type of string does not bear a topological charge, but only magnetic flux. For an open spatial string, however, $j^{0} \neq 0$ and we see that it carries topological charge but no magnetic flux. Notice that, in this case, $C$ is still closed in spite of the fact that the string is open.

In what follows, we are going to study the operators creating the quantum states corresponding to the above mentioned field configurations. For this, we will need the basic commutation rules for the theory described above, which are given by the expression

$$
\begin{aligned}
& {\left[B^{i j}(\vec{x}, t), A^{k}(\vec{y}, t)\right]=i \epsilon^{i j k} \delta(\vec{x}-\vec{y}),} \\
& {\left[B^{i j}, B^{k l}\right]=\left[A^{i}, A^{j}\right]=0 .}
\end{aligned}
$$

after elimination of the second-class constraints of the theory (no gauge condition was used).

\section{THE MAGNETIC FIELD AND TOPOLOGICAL CHARGE OPERATORS}

Let us determine now the creation operators for the field configurations corresponding to the closed string and point topological charge. Firstly, we shall obtain the creation operator for the magnetic field associated to a closed string at the spacelike curve $C$. Following [4, 8], we write

$$
\sigma(C, t)=\exp \left\{\frac{i a}{2} \int_{S(C)} d^{2} \xi^{i j} B^{i j}(\vec{\xi}, t)\right\}
$$

where $d^{2} \xi^{i j}(i, j$ along $S(C))$ is the surface element of $S(C)$, an arbitrary surface with border along $C$.

According to the field equation (2.5), the string is associated to a magnetic flux along $C$. In order to characterize $\sigma(C)$ as a quantum creation operator for the field configuration associated to the string, let us evaluate $\left[\mathcal{B}^{i}, \sigma(C)\right]$. For this purpose, we write $\sigma(C) \equiv e^{\alpha(C)}$ and, using (2.7), obtain

$$
\left[\mathcal{B}^{i}(\vec{x}, t), \alpha(C)\right]=a \oint_{C} d \xi^{i} \delta(\vec{\xi}-\vec{x})
$$

From this we get

$$
\left[\mathcal{B}^{i}(\vec{x}, t), \sigma(C, t)\right]=\left[a \oint_{C} d \xi^{i} \delta(\vec{\xi}-\vec{x})\right] \sigma(C, t),
$$

which implies

$$
\mathcal{B}^{i}(\vec{x})\left|\sigma(C)>=\left[a \oint_{C} d \xi^{i} \delta(\vec{\xi}-\vec{x})\right]\right| \sigma(C)>,
$$

which clearly shows that the operator $\sigma$ indeed creates an eigenstate of the magnetic field with the correct eigenfield-configuration.

We now turn to the topological charge creation operator. Let us consider

$$
\mu(\vec{x}, t)=\exp \left\{-i b \int_{-\infty, L}^{\vec{x}} d \xi^{i} A^{i}(\vec{\xi}, t)\right\},
$$

where $L$ is an arbitrary line going from $-\infty$ to $\vec{x}$. We are going to evaluate the commutator $[Q, \mu]$, where

$$
Q=\int d^{3} x \epsilon^{i j k} \partial_{i} B_{j k}
$$

is the topological charge operator. Writing $\mu(\vec{x}, t) \equiv$ $e^{\beta}(\vec{x}, t)$ and using $(2.7)$, we obtain

$$
[Q, \beta(\vec{y}, t)]=b \int d^{3} x \delta(\vec{x}-\vec{y}),
$$

which implies $[Q, \mu]=b \mu$ or $Q|\mu>=b| \mu>$, thus showing that the operator $\mu$ carries $b$ units of topological charge.

According to our considerations after Eq. (2.6), observe that we may consider (3.5) as the creation operator for the field configuration associated to an open string at the curve $L$, which bears a topological charge at its extremity $\vec{x}$. 


\section{GENERALIZED STATISTICS OF COMPOSITE STRINGS WITH TOPOLOGICAL CHARGE AND MAGNETIC FIELD}

Before studying the commutation rules of the composite topologically charged string operators, let us determine the dual algebra satisfied by the magnetic field and topological charge creation operators, $\sigma \equiv e^{\alpha}$ and $\mu \equiv e^{\beta}$, given respectively by (3.1) and (3.5). Using (2.7), we obtain

$$
[\alpha(C, t), \beta(\vec{y}, t)]=i a b \int_{S(C)} d^{2} \xi^{i} \int_{-\infty, L}^{\vec{y}} d \eta^{i} \delta(\vec{\xi}-\vec{\eta})
$$

where $d^{2} \xi^{i} \equiv \frac{1}{2} \epsilon^{i j k} d^{2} \xi^{i j}$.

We now use the identity

$$
\partial_{(\xi)}^{i}\left[\frac{1}{4 \pi|\vec{\xi}-\vec{x}|}\right]= \begin{cases}\epsilon^{i j k} \partial_{(\xi)}^{j} \varphi_{k}(\vec{\xi}-\vec{x}) ; & \vec{\xi} \notin V_{L} \\ \int_{-\infty, L}^{\vec{x}} d \eta^{i} \delta(\vec{\eta}-\vec{\xi}) ; & \vec{\xi} \in V_{L}\end{cases}
$$

where $V_{L}$ is a cone of infinitesimal angle with vertex at $\vec{\xi}=\vec{x}$ and axis along the line $L:(-\infty, \vec{x})$ and $\vec{\varphi}=$ $\frac{1-\cos \theta}{r \sin \theta} \hat{\varphi}$, with $r=|\vec{\xi}-\vec{x}|$. Because of the delta-function, observe that (4.1) is non vanishing only inside $V_{L}$, hence we can use the second part of 4.2 to write

$$
\begin{aligned}
{[\alpha(C, t), \beta(\vec{y}, t)] } & =-i \frac{a b}{4 \pi} \int_{S(C)} d^{2} \xi^{i} \frac{(y-\xi)^{i}}{|\vec{y}-\vec{\xi}|^{3}} \\
& =-i \frac{a b}{4 \pi} \Omega(\vec{y} ; C)
\end{aligned}
$$

where $\Omega(\vec{y} ; C)$ is the solid angle comprised between $\vec{y}$ and the curve $C$. Since $[\alpha, \beta]$ is a c-number, it immediately follows that

$$
\sigma\left(C_{x}, t\right) \mu(\vec{y}, t)=\mu(\vec{y}, t) \sigma\left(C_{x}, t\right) \exp \left\{-i \frac{a b}{4 \pi} \Omega(\vec{y} ; C)\right\} .
$$

This is the dual algebra [9, 10] satisfied by the magnetic string field and topological charge operators, which is identical to the one satisfied by the corresponding operators in the B-F theory [4].

We now consider the composite operator creating a magnetic-field-bearing-topologically-charged string. Choosing the string as a circle $C_{x}$ centered at $\vec{x}$, this may be defined as

$$
\psi\left(x ; C_{x} ; t\right)=\lim _{\vec{x} \rightarrow \vec{y}} \mu(\vec{x}, t) \sigma\left(C_{y}, t\right)
$$

Now, considering the property $\Omega\left(\vec{x} ; C_{y}\right)-\Omega\left(\vec{y} ; C_{x}\right)=$ $4 \pi \epsilon\left(\Omega\left(\vec{x} ; C_{y}\right)\right)$, where $\epsilon(x)$ is the sign function, we get

$$
\begin{aligned}
& \psi\left(x ; C_{x} ; t\right) \psi\left(y ; C_{y} ; t\right) \\
& \quad=e^{-i a b \epsilon\left(\Omega\left(\vec{x} ; C_{y}\right)\right)} \psi\left(y ; C_{y} ; t\right) \psi\left(x ; C_{x} ; t\right) .
\end{aligned}
$$

This commutation relation shows that the composite field operator $\psi$, which creates the quantum field states with topological charge and magnetic flux, associated to the composite of a closed and an open string, possesses generalized statistics $S=\frac{a b}{2 \pi}$ in the four- dimensional ChernSimons theory given by (2.3). Suppose now the quantum bosonic string, Nambu-Goto for instance, is created by a certain bosonic operator $\Sigma$, in the absence of the CS coupling. When the string is coupled to the CS Lagrangian, the appropriate operator becomes $\Sigma \sigma$ or $\Sigma \mu$ (where $\sigma$ and $\mu$ are given by (3.1) and (3.5)), respectively, according to whether the string is closed or open. Hence, the appropriate operator for the composite closed-open string quantum states becomes $\Sigma_{C} \sigma \Sigma_{O} \mu=\Sigma_{C} \Sigma_{O} \psi$. This has generalized statistics $S$, according to (4.6). The complete description of the statistical transmutation of bosonic strings coupled to CS theory in $3+1 \mathrm{D}$, therefore, has been achieved at a full quantum level.

\section{REMARKS ON THE FUNCTIONAL INTEGRAL FORMULATION}

In a previous work [1], it has been shown that a bosonic string coupled to four-dimensional Chern-Simons theory as in (2.3), with $j^{\mu}$ given by (2.6) undergoes Fermi-Bose transmutation. The demonstration is made within the functional integral formulation, where a sum is performed upon all possible universe-sheets $S(C)$ associated to $C$. We would like to remark that the result found in $[\mathbb{1}]$ is related to ours in an interesting way. In order to see this, we note that the coupling with $A_{\mu}$ in 2.3 involves a sum over all values of the index $\mu$ and this fact is used in the derivation of the fermionic algebra that leads to the Fermi-Bose transmutation in [1]. Observe now that the spacelike part of the sums corresponds to a closed spatial string, which, as we saw, bears a magnetic flux along it. The timelike part of the sums, on the other hand, correspond to an open spatial string, which bears a point topological charge at its tip, as we have seen. The whole sum in (2.3), therefore, comprises the composite state of a topologically charged open string and a magnetic flux carrying closed string, which is precisely the situation we have considered here from the operator point of view. Transmutation to a generalized statistics should be also obtained in the framework used in [1] by considering arbitrary parameters in the couplings in (2.3). We see that the present work, therefore, unifies the results obtained in [1] and [2] and opens the possibility of evaluation of quantum correlators for the operators describing the quantized field associated to strings coupled to vector and tensor fields $A_{\mu}$ and $B_{\mu \nu}$. 


\section{Acknowledgments}

This work was supported in part by CNPq, FAPERJ and PRONEX-66.2002/1998-9

[1] X. Fustero, R. Gambini and A. Trias, Phys. Rev. Lett. 62, 1964 (1989).

[2] R. Gambini and R. Setaro, Phys. Rev. Lett. 65, 2623 (1990).

[3] H. Fort and R. Gambini, Phys. Lett. B372, 226 (1996).

[4] E.C. Marino, Phys. Lett. B393, 383 (1997).

[5] J. Barcelos-Neto and E.C. Marino, Europhys. Lett. 57, 473 (2002).

[6] J. Barcelos-Neto and E.C. Marino, work in progress.

[7] E. Cremer and J. Scherk, Nucl. Phys. B72, 117 (1974).
[8] E.C. Marino, Phys. Rev. D53, 1001 (1996).

[9] L.P. Kadanoff and H. Ceva, Phys. Rev. B3, 3918 (1971); G. 't Hooft, Nucl. Phys. B138, 1 (1978).

[10] E.C. Marino and J.A. Swieca, Nucl. Phys. B170 [FS1], 175 (1980); E.C. Marino, B. Schroer and J.A. Swieca, Nucl. Phys. B200 [FS4], 499 (1982); E.C. Marino, Phys. Rev. D38, 3194 (1988); E.C. Marino and J.E. Stephany Ruiz, Phys. Rev. D39, 3690 (1989). 